\title{
Direct Torque Control of Induction Motor With Fuzzy Stator Resistance Adaptation
}

\author{
F. Zidani, D. Diallo, Member, IEEE, M. E. H. Benbouzid, Senior Member, IEEE, and R. Naït-Saïd
}

\begin{abstract}
A new stator resistance estimator using fuzzy logic is proposed. The input variables of the fuzzy logic identifier are the input and output of the low-pass (LP) filter used to integrate the back-emf. Simulation results, using Matlab-Simulink, comparing the fuzzy estimator and a classical integrator in a direct torque control (DTC) scheme prove the superiority of the novel approach. The stator flux locus is smoothed and therefore torque ripples are reduced.
\end{abstract}

Index Terms-Direct torque control, fuzzy logic, induction motor, stator resistance.

\section{INTRODUCTION}

D IRECT torque control (DTC) based only on stator flux estimation using line current measurement and terminal voltages measurement or reconstruction, from the switching states and the dc bus voltage, is an alternative to field oriented control (FOC). Conventional DTC [1] requires no mechanical sensor, no current regulator, no coordinate transformation, and depends only on stator resistance. Because of its good dynamic performances and the robustness, it has been widely used despite the inherent drawbacks (e.g., variable switching frequency, high torque ripples at low speed). Many papers have been published to improve the DTC capability with speed sensorless design [2] or torque ripple reduction. One of the main problems is the drive behavior at low speed or standstill where stator flux estimation becomes strongly dependant on the stator resistance.

In this paper, a fuzzy stator resistance identifier is introduced without any additional measurement like temperature or any other control method like observers or estimators, which are computational time consuming and delicate to tune. The fuzzy logic identifier (FLI) uses the LP filter input and output to compute the input variable.

\section{DTC BASIC PRINCIPLES}

The basic DTC strategy is built upon the direct control of stator flux and stator voltage. The stator flux estimation based on the induction motor voltage model in the stator flux reference frame is determined from

$$
\bar{V}_{\mathrm{s}}=R_{\mathrm{s}} \bar{I}_{\mathrm{s}}+j \frac{d \bar{\phi}_{\mathrm{s}}}{d t} .
$$

Manuscript received July 12, 2005; revised November 25, 2005. Paper no. TEC-PESL-00062-2005.

F. Zidani and R. Naït-Saïd are with the LSPIE, University of Batna, Batna, Algeria.

D. Diallo is with the LGEP, University of Paris XI, France.

M. E. H. Benbouzid is with the LIME, University of Western Brittany, Brest, France.

Digital Object Identifier 10.1109/TEC.2006.874251

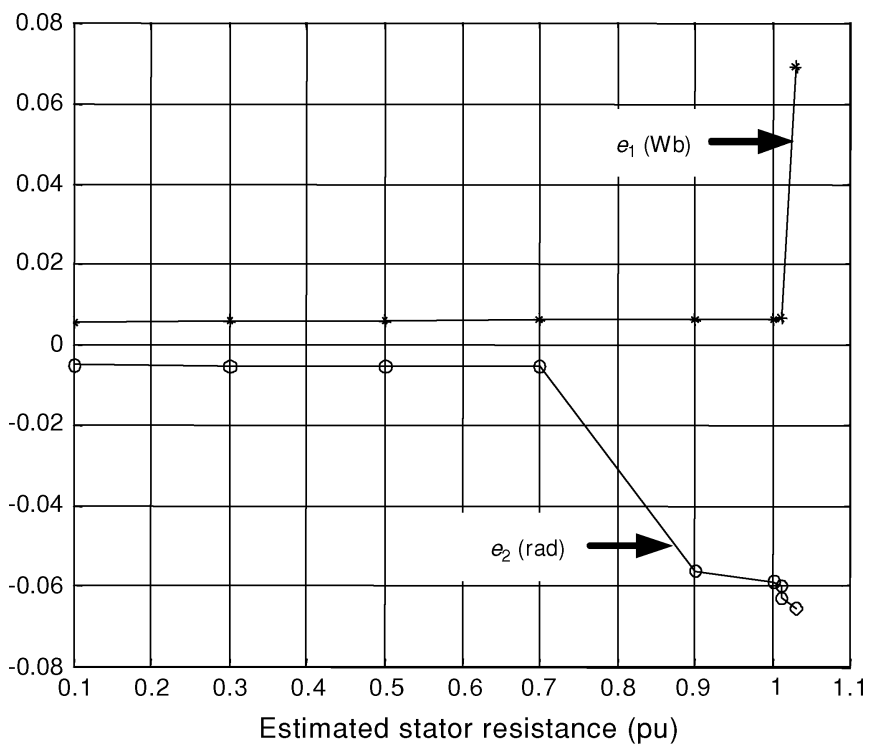

Fig. 1. Errors $e_{1}$ and $e_{2}$ variation versus estimated stator resistance.

The stator flux space vector $\phi_{\mathrm{s}}$ is estimated by integrating the motor back-emf space vector.

$$
\hat{\bar{\phi}}_{\mathrm{s}}=\int\left(\bar{V}_{\mathrm{s}}-\hat{R}_{\mathrm{s}} \bar{I}_{\mathrm{s}}\right) d t
$$

The stator flux estimator from (2) becomes critical at low speed. To achieve good performance at standstill and at low speed, integration drift due to the dc offset or measurement noise must be eliminated by the introduction of a low-pass filter with a cutoff frequency $\omega_{\mathrm{f}}$ [3]. The flux estimator output becomes

$$
\bar{\phi}_{\mathrm{sf}}=\frac{\bar{V}_{\mathrm{s}}-R_{\mathrm{s}} \bar{I}_{\mathrm{s}}}{j\left(\omega_{\mathrm{s}}-\omega_{\mathrm{f}}\right)} .
$$

At low speed, the resistive term becomes preponderant, and therefore the stator resistance needs to be well known. Indeed, if the estimated resistance is greater than the actual one, the time constant of the following differential equation, obtained from (1), becomes negative, leading to instability.

$$
\frac{d \bar{\phi}_{\mathrm{s}}}{d t}+\frac{\left(R_{\mathrm{s}}-\hat{R}_{\mathrm{s}}\right)}{\sigma L_{\mathrm{s}}} \bar{\phi}_{\mathrm{s}}=\frac{d \hat{\bar{\phi}_{\mathrm{s}}}}{d t}+\frac{L_{m}\left(R_{\mathrm{s}}-\hat{R}_{\mathrm{s}}\right)}{\sigma L_{\mathrm{s}} L_{r}} \bar{\phi}_{r} .
$$

\section{Fuzzy Logic Based Stator Resistance IDENTIFICATION SCHEME}

This approach is based on the phase and magnitude errors between the estimated flux and the filtered one. The FLI input variables are the stator flux magnitude and the phase errors. For 


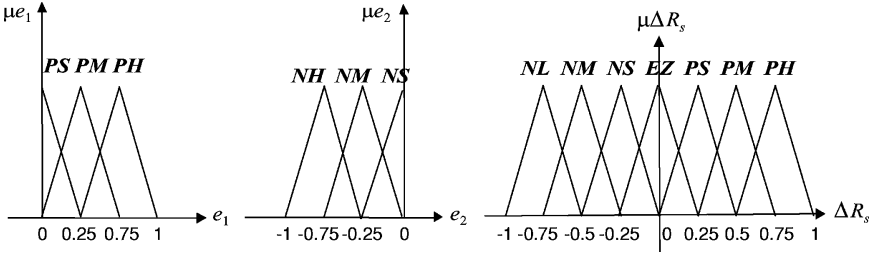

Fig. 2. Input/output variables membership functions.

TABLE I

FLI LINGUISTIC RULES

\begin{tabular}{|c|c|c|c|}
\hline$e_{e_{2}} e^{e_{1}}$ & $P S$ & $P M$ & PH \\
\hline$N S$ & $E Z$ & $P S$ & $P H$ \\
\hline$N M$ & $N S$ & $N M$ & $P H$ \\
\hline $\mathrm{NH}$ & $P S$ & $P M$ & $P H$ \\
\hline
\end{tabular}

(NH: Negative High, NM: Negative Medium, NS: Negative Small, PS: Positive Small, PM: Positive Medium, PH: Positive High)

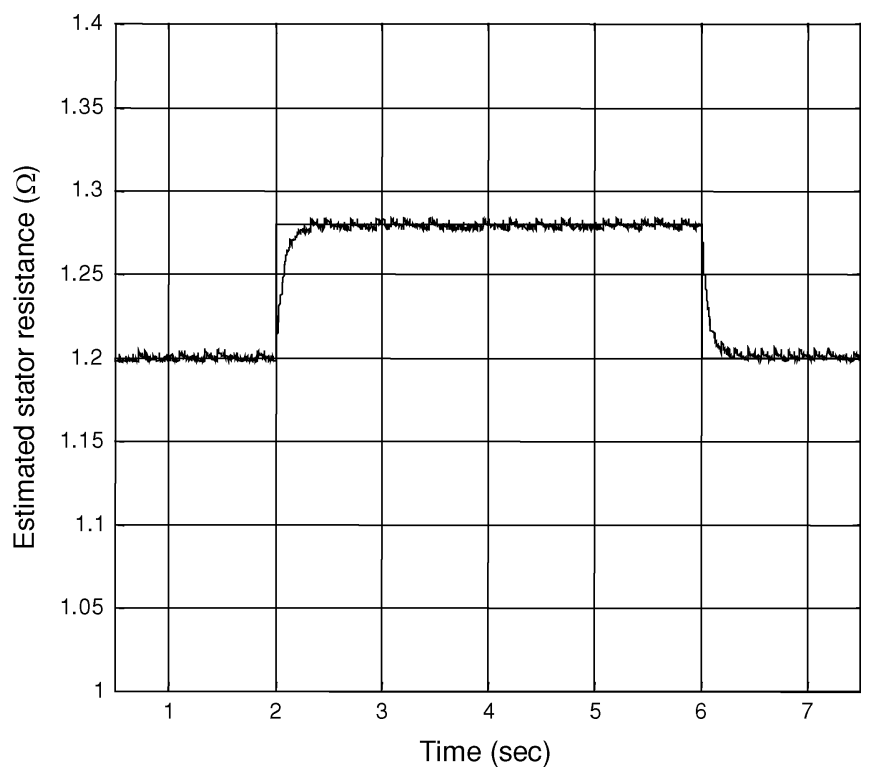

Fig. 3. Stator resistance estimations.

the $k$ th sampling interval we have

$$
\left\{\begin{array}{l}
e_{1}(k)=\left|\bar{\phi}_{\mathrm{s}}(k)\right|-\left|\bar{\phi}_{\mathrm{sf}}(k)\right| \\
e_{2}(k)=\operatorname{phase}\left(\bar{\phi}_{\mathrm{sf}}\right)-\operatorname{phase}\left(\bar{\phi}_{\mathrm{s}}\right)
\end{array} .\right.
$$

The FLI output variable is the rate of change $\Delta R_{\mathrm{S}}$ that is generated through fuzzy inference and defuzzification. The crisp output $\Delta R_{\mathrm{S}}(k)$ is integrated in such a way that the estimated stator resistance is given by

$$
\hat{R}_{\mathrm{s}}(k)=\hat{R}_{\mathrm{s}}(k-1)+\Delta \hat{R}_{\mathrm{s}}(k) .
$$

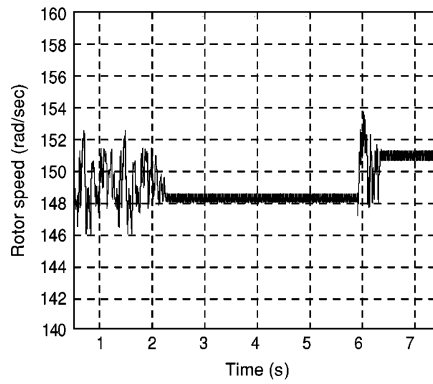

(a)

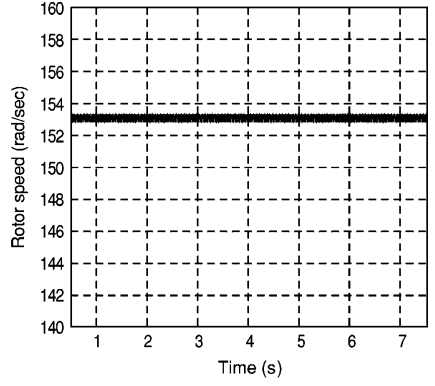

(b)
Fig. 4. Steady-state speed response: (a) without FLI and (b) with FLI.

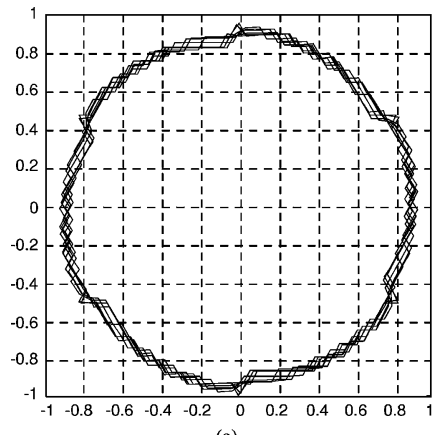

(a)

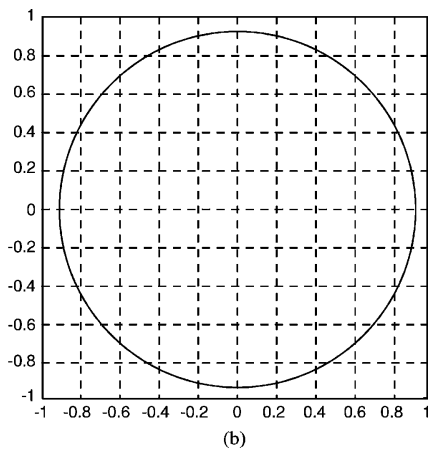

(b)
Fig. 5. Stator flux locus: (a) without FLI and (b) with FLI.

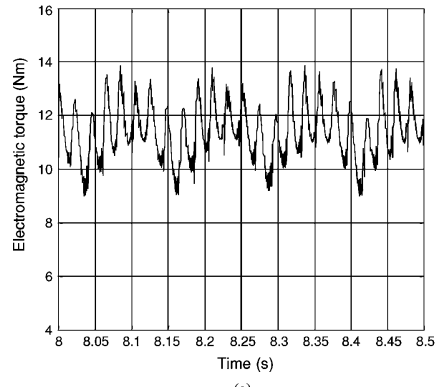

(a)

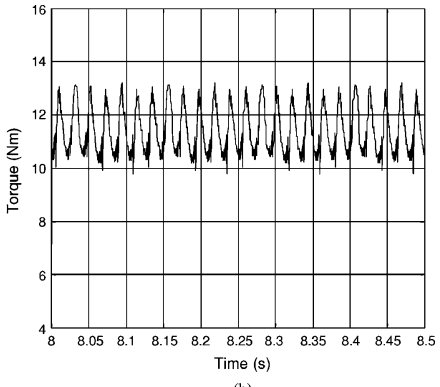

(b)
Fig. 6. Steady-state torque response: (a) without FLI and (b) with FLI.

\section{FLI DESIGN}

Fig. 1 shows the evolution of both errors $e_{1}$ and $e_{2}$ under typical detuning conditions. The current sensors are supposed ideal. The membership functions of the normalized input/output variables are given in Fig. 2. With two inputs and three membership values, the estimator contains nine rules that are listed in Table I.

\section{PERformanCES OF THE IMPROVED DTC}

To show the performance of the proposed FLI, a step change in $R_{\mathrm{S}}$ has been applied in steady state. As illustrated by Fig. 3, the estimator converges to the actual value. As long as the estimated value is lower than the actual resistance, the stability of the control strategy is maintained. In Fig. 4(a) we can notice that the stator resistance error causes improper flux estimation making the DTC perform poorly. The results displayed in Fig. 5 
show the stator flux improvement. The trajectory is smoothened, and therefore torque ripple and line current harmonic content reduction is expected. This is confirmed by the torque curves in Fig. 6. Furthermore, the dynamic behavior of the DTC during load change is not modified. The estimator is sampled at a higher rate and the stator resistance real evolution is not abrupt as it is shown in Fig. 3 for example.

\section{CONCLUSION}

The performances of a DTC scheme with a stator resistance fuzzy identification have been analyzed. The proposed fuzzy estimator improves the stator flux estimation accuracy leading to a smooth trajectory and therefore reducing the torque ripples. This estimator is particularly suited in applications needing high torque at low speed and improves the performance of control strategy in applications where thermal impact on resistance variation is no more negligible.

\section{APPENDIX}

\section{RATED DATA OF THE SIMUlated INDUCTION MOTOR}

Rated Values: 4 kW, $50 \mathrm{~Hz}, 220 / 380 \mathrm{~V}(\Delta / \mathrm{Y})$, 15/8.6 A $(\Delta / \mathrm{Y}), 1440 \mathrm{rpm}$, pole pair (2), Power factor $(0.8)$.

Parameters \& Constants: $R_{\mathrm{s}}=1.200 \Omega, R_{r}=1.800 \Omega, L_{\mathrm{s}}=$ $0.156 \mathrm{H}, L_{r}=0.156 \mathrm{H}, M=0.143 \mathrm{H}, J=0.024 \mathrm{~kg} \cdot \mathrm{m}^{2}, f=$ 0.011 IS.

\section{REFERENCES}

[1] G. S. Buja and M. P. Kazmierkowski, "Direct torque control of PWM inverter-fed ac motors-A survey," IEEE Trans. Ind. Electron., vol. 51, no. 4, pp. 744-757, Aug. 2004.

[2] C. Lascu and A. M. Trzynadlowski, "A sensorless hybrid DTC drive for high-volume low-cost applications," IEEE Trans. Ind. Electron., vol. 51, no. 5, pp. 1048-1055, Oct. 2004.

[3] N. R. N. Idris and A. H. M. Yatim, "An improved stator flux estimation in steady-state operation for direct torque control of induction machines," IEEE Trans. Ind. Appl., vol. 38, no. 1, pp. 110-116, Jan.-Feb. 2002. 